\section{Vulnerabilidade de homens que fazem sexo com homens no contexto da AIDS}

\author{
Vulnerability of men who have sex with men \\ in the context of AIDS
}

\footnotetext{
${ }^{1}$ Universidade Federal de Mato Grosso do Sul, Campo Grande, Brasil.

2 Universidade para o Desenvolvimento do Estado e da Região do Pantanal, Campo Grande, Brasil.

Correspondência S. M. O. Andrade Universidade Federal de Mato Grosso do Sul. Rua Luis Freire Benchetrit 258, Campo Grande, MS 79004-500, Brasil. anart.msi@terra.com.br
}

\begin{abstract}
The number of AIDS cases in men who have sex with men (MSM) decreased in the initial years of the epidemic, but the percentage of cases in this group has remained unaltered in the last five years. This study, aimed to improve knowledge on the impact of AIDS in the daily lives of MSM, was conducted in Campo Grande, Mato Grosso do Sul State, Brazil, using a questionnaire and interviews. The results show that MSM perceive their vulnerability to HIV infection, referring to inconsistent condom use and multiple sex partners, given that $65 \%$ of homosexuals, $75 \%$ of bisexuals, and $33 \%$ of transvestites reported up to 5 partners in the previous month and emphasizing that 59\% of the group reported more than 11 partners during the same period. The susceptibility of certain groups and the identification and understanding of their specificities pose a major challenge in any geographic context where such conditions are present.
\end{abstract}

Male Homosexuality; Acquired Immunodeficiency Syndrome; Sex Behavior
Sonia Maria Oliveira de Andrade 1

Edson Mamoru Tamaki 1

Joaquim Miguel Vinha 2

Mauricio Antonio Pompilio 2

Cybele Weber Prieto ${ }^{2}$

Laura Moreno de Barros 2

Livia Beraldo de Lima 2

Marina de Capua Chaguri 2

Socorro Andrade de Lima Pompilio 2

\section{Introdução}

Os dados do Ministério da Saúde 1 mostram que os números de AIDS em população de homens que fazem sexo com homens, quando se considera a transmissão sexual, permanecem estabilizados em patamares elevados e isso provavelmente se dá como conseqüência de sua vulnerabilidade.

Examinar os significados subjetivos da sexualidade, a esfera do comportamento, requer que seja feita, além da análise do corpo com suas funções e sensações, a consideração do sentido e do papel da sexualidade no interior da nossa cultura 2 .

A sexualidade é enquadrada por um conjunto de leis, costumes, regras e normas variáveis no tempo e no espaço, sendo um fenômeno socialmente construído ${ }^{3}$. Assim, as maneiras pelas quais as identidades sexuais são formadas e transformadas em relações de poder e dominação que podem moldar e estruturar as interações sexuais devem ser levadas em consideração no desenvolvimento de estratégias mais eficazes para a prevenção da AIDS 4,5.

É patente o elevado grau de informação que possuem os homens que fazem sexo com homens (HSH) tanto sobre a doença quanto sobre os modos de transmissão, mas também é marcante a defasagem entre o conhecimento e a adoção do sexo seguro, repercutindo na manutenção elevada de casos notificados de AIDS nesse grupo. No 
Brasil, 22,5\% dos casos de AIDS encontram-se em pessoas pertencentes às categorias de identidade bissexual e homossexual, e em Mato Grosso do Sul 35\% dos casos se dão nessa população 1,6.

No contexto da AIDS, a mudança de comportamento tem demonstrado ser a forma mais efetiva de diminuir o avanço do vírus 7 , mas a mudança de comportamento de HSH continua a ser o grande desafio.

\section{Procedimentos metodológicos}

Em razão da inexistência de estudos com HSH em Campo Grande, Mato Grosso do Sul, a presente pesquisa configura-se como exploratória, buscando-se a caracterização inicial da questão para a posterior identificação de possíveis novos aspectos a serem profundamente investigados. Considerando a impossibilidade de identificação quantitativa da população de HSH em Campo Grande, decidiu-se pela amostra por conveniência, recrutada por meio da estratégia de captação de sujeitos em rede, com base na localização de informantes-chave.

Por intermédio dos primeiros sujeitos investigados, foi feito contato com entidades que agregam HSH, quais sejam: Associação dos Travestis de Campo Grande (ATMS), Centro de Protagonismo Juvenil (CPJ), Grupo Iguais e o Grupo de Estudo de Livres Sexualidades (GELLS), da Universidade Federal de Mato Grosso do Sul. Foram feitas coletas, ainda, na Praça Ari Coelho, região central de Campo Grande, e em um bar onde os HSH se reúnem. Foram aplicados questionários em 52 sujeitos, sendo 34 homossexuais, 12 travestis, 4 bissexuais e 2 garotos de programa.

O questionário, composto de perguntas fechadas e mistas, possibilitou a identificação da autopercepção do comportamento sexual, do uso de preservativos, da atividade sexual e da quantidade de parceiros sexuais.

A pesquisa obteve a aprovação de Comitê de Ética em Pesquisa Envolvendo Seres Humanos por haver atendido as recomendações da Resolução n. 196/96 do Conselho Nacional de Saúde, incluindo o Termo de Consentimento Livre e Esclarecido individual.

\section{Resultados e discussão}

Tomando por referência a totalidade dos HSH participantes da pesquisa, foram inicialmente analisadas as questões referentes à autopercepção do comportamento sexual de risco e uso do preservativo. Constata-se que apenas $27 \%$ dos entrevistados assumiram ter, atualmente, com- portamento de risco. Tais dados são inconsistentes quando se promove o confronto com informações referentes ao uso do preservativo e ao número de parceiros.

Quanto ao uso do preservativo foram oferecidas quatro possibilidades de resposta referente à constância do uso, quais sejam: em todas as relações sexuais; apenas com parceiros eventuais; às vezes; e nunca.

Apesar de 73\% dos entrevistados não se considerarem como expostos atualmente ao risco de contrair o HIV, 35\% afirmam não fazer uso do preservativo em todas as relações sexuais. Considerando que as principais correntes referentes à autoproteção defendem que o comportamento preventivo se relaciona com a vulnerabilidade percebida, os resultados desta pesquisa indicam que a validade dessa posição, no que se refere ao HIV, necessitaria ser melhor investigada 8 .

As falhas ou inconsistências relacionadas ao uso do preservativo por parte da população entrevistada são similares as de vários estudos realizados no Brasil e em outros países como o realizado com a população homo e bissexual americana ${ }^{9}$ e mexicana 10 , com destaque ao uso mais consistente por parte da população bissexual.

Outros elementos relevantes no risco para a aquisição de DST e para a infecção pelo HIV são o início precoce da vida sexual e o número de parcerias sexuais na vida 11 .

A idade média de início de relacionamento sexual de $17 \%$ dos entrevistados se situou por volta dos oito anos; $44 \%$ o fizeram por volta dos 13 anos de idade, o que se configura como um importante elemento favorável à aquisição de DST, incluindo a AIDS.

A maioria (79\%) iniciou sua vida sexual com parceiro do sexo masculino, cabendo ressaltar que essa foi a forma de início da vida sexual de todos os travestis e michês.

A questão da quantidade de parceiros configura-se como primordial, e na análise da Tabela 1 é possível perceber que tanto os travestis como os michês se diferenciam dos bissexuais e dos homossexuais, considerando a quantidade de parceiros sexuais no último mês. Aproximadamente $43 \%$ dos travestis tiveram mais de vinte parceiros no último mês, o que, aliado ao fato de haver uso descontinuado de preservativo, tornaos extremamente vulneráveis ao HIV e às DST.

Sabe-se que há uma associação entre HIV e o número de parceiros sexuais 12,13 , e tal fato não é desconhecido pelo grupo, visto que $75 \%$ dos travestis consideram-se como tendo comportamento atual de risco.

A múltipla parceria é considerada como um fator de risco quando o intercurso sexual, seja anal seja vaginal, se dá sem o uso do preservativo 13 . 
Distribuição de homens que fazem sexo com homens por categoria, segundo o número de parceiros sexuais no último mês. Campo Grande, Mato Grosso do Sul, Brasil, 2004 ( $n=52$ ).

\begin{tabular}{|c|c|c|c|c|c|c|c|c|}
\hline \multirow[b]{2}{*}{ Parceiros } & \multicolumn{2}{|c|}{ Homossexual $(n=34)$} & \multicolumn{2}{|c|}{ Bissexual $(n=4)$} & \multicolumn{2}{|c|}{ Travesti $(n=12)$} & \multicolumn{2}{|c|}{ Michê $(n=2)$} \\
\hline & $\mathrm{n}$ & $\%$ & $\mathbf{n}$ & $\%$ & $\mathrm{n}$ & $\%$ & $\mathbf{n}$ & $\%$ \\
\hline Nenhum & 5 & 15 & 1 & 25 & 1 & 8 & 0 & 0 \\
\hline 1 & 15 & 45 & 1 & 25 & 3 & 25 & 0 & 0 \\
\hline $2-5$ & 7 & 20 & 2 & 50 & 1 & 8 & 0 & 0 \\
\hline $6-10$ & 3 & 8 & 0 & 0 & 0 & 0 & 2 & 100 \\
\hline $11-20$ & 2 & 6 & 0 & 0 & 2 & 16 & 0 & 0 \\
\hline Mais de 20 & 2 & 6 & 0 & 0 & 5 & 43 & 0 & 0 \\
\hline Total & 34 & 100 & 4 & 100 & 12 & 100 & 2 & 100 \\
\hline
\end{tabular}

Assim, mais uma vez destaca-se a maior vulnerabilidade dos travestis.

Foi perguntado aos entrevistados se haviam tido relação sexual com pessoa sabidamente portadora do HIV, já que o status sorológico dos parceiros, tendo em vista o sexo desprotegido, caracteriza um alto fator de vulnerabilidade para o HIV.

Dos entrevistados, sete (13\%) referiram ter se relacionado sexualmente com parceiros HIV+, sendo quatro homossexuais e três travestis.

Dentre os quatro homossexuais, apenas dois afirmaram que houve uso de preservativo na relação sexual, o mesmo sendo verdadeiro para dois travestis. Vale destacar que quando há medo e receio, estes fatores influenciam diretamente na estimativa do próprio risco e no comportamento sexual, que se torna mais seguro ${ }^{14}$. Destaca-se a baixa preocupação com a própria saúde, evidenciada pela exposição dos HSH ao risco de se infectar pelo HIV e de contraírem outras DST.

\section{Conclusão}

Os resultados da pesquisa levantam aspectos cujo conhecimento deveria ser aprofundado para subsidiar intervenções que visem à redução de comportamentos de risco de homens que fazem sexo com homens.

A responsabilidade no sentido da mudança da realidade atual extrapola o âmbito da saúde e não isenta as autoridades de contribuições importantes a fim de que sejam desenvolvidas ações que privilegiem comunidades específicas e que sejam adequadas a determinados grupos, como os homens que fazem sexo com homens em suas diferentes categorias e, em particular, os travestis, conforme apontam os resultados da presente pesquisa.

A suscetibilidade de determinados grupos e a identificação e compreensão de suas particularidades constituem, ainda, um grande desafio a ser enfrentado não só em Campo Grande, mas em qualquer espaço geográfico em que essas condições se façam presentes. 


\section{Resumo}

O número de casos de AIDS entre homens que fazem sexo com homens (HSH) diminuiu nos primeiros anos da epidemia, mas nos últimos cinco anos os percentuais de casos de AIDS nessa população permanecem inalterados. A pesquisa, que teve por objetivo aprofundar a compreensão sobre o impacto da AIDS no cotidiano dos HSH, foi realizada em Campo Grande, Mato Grosso do Sul, Brasil, utilizando-se questionário e entrevistas. Os resultados mostram que os HSH percebem-se como vulneráveis à infecção pelo HIV, referem uso inconsistente do preservativo e ocorrência de múltiplas parcerias, visto que 65\% dos homossexuais, 75\% dos bissexuais e 33\% dos travestis tiveram até cinco parceiros no último mês, destacando-se que 59\% deste grupo tiveram mais de 11 parceiros no mesmo período. A suscetibilidade de determinados grupos e a identificação e compreensão de suas particularidades constituem o grande desafio a ser enfrentado em qualquer espaço geográfico em que essas condições se façam presentes.

Homossexualidade Masculina; Síndrome de Imunodeficiência Adquirida; Comportamento Sexual

\section{Referências}

1. Ministério da Saúde. AIDS: dados do Brasil. Boletim Epidemiológico da AIDS. http://www.aids.gov. br/final/biblioteca/Boletim_dezembro_2003/Brasil.asp (acessado em 20/Out/2004)

2. Duarte LFD. O império dos sentidos: sensibilidade, sensualidade e sexualidade na cultura ocidental moderna. In: Heilborn ML, organizadora. Sexualidade: o olhar das ciências sociais. Rio de Janeiro: Jorge Zahar Editor; 1999. p. 21-30.

3. Lhomond B. Sexualidade e juventude na França. In: Heilborn ML, organizador. Sexualidade: o olhar das ciências sociais. Rio de Janeiro: Jorge Zahar Editor; 1999. p. 77-95.

4. Parker R. Sexo entre homens: consciência da AIDS e comportamento sexual entre os homens homossexuais e bissexuais no Brasil. In: Parker RG, Bastos C, Galvão J, Pedrosa JS, organizadores. A AIDS no Brasil. Petrópolis: Editora Relume-Dumará; 1994. p. 129-49.

5. Mansergh G. Paradigm shift for HIV prevention in the United States. AIDSci 2002; 2(10). http://aidscience.com/Articles/AIDScienceo21.asp (acessado em 19/Out/2004).

6. Secretaria de Estado de Saúde do Mato Grosso do Sul. Série histórica DST/Aids. Boletim Epidemiológico 2002.

7. Pinkerton SD, Abramson PR. The joys of diversification: vaccines, condoms, and AIDS prevention. AIDS Public Policy J 1995; 10:148-56.

\section{Colaboradores}

Todos os autores participaram, integral ou parcialmente, de cada uma das fases de desenvolvimento da pesquisa.

\section{Agradecimentos}

Pesquisa financiada pelo Departamento de Ciência e Tecnologia, Secretaria de Ciência, Tecnologia e Insumos Estratégicos, Ministério da Saúde e Fundação de Apoio ao Desenvolvimento do Ensino, Ciência e Tecnologia do Estado do Mato Grosso do Sul (Termo de Outorga 081/03).

8. Gerrade M, Gibbons FC, Bushman BJ. Relation between perceived vulnerability to HIV and precautionary sexual behavior. Psychol Bull 1996; 119:390-409.

9. Katz MH, Schwarcz SK, Kellogg TA, Klausner JD, Dilley JW, Gibson S, et al. Impact of highly active antiretroviral treatment on HIV seroincidence among men who have sex with men: San Francisco. Am J Public Health 2002; 92:388-94.

10. Izazola-Licea JA, Pulerwitz J, Gortmarker SL. Extrarelational sex among Mexican men and their partners risk of HIV and other sexually transmitted diseases. Am J Public Health 2001; 91:1650-2.

11. Malloy TE, FisherWA, Albright L, Misovich SJ, Fisher JD. Interpersonal perception of the opposite sex. Health Psychol 1997; 16:480-6.

12. Aispinwall LG, Kemeny ME, Taylor SE, Schneider SG, Dudley JP. Psychosocial predictors of gay men's AIDS risk-reduction behavior. Health Psychol 1991; 10:432-44

13. Gerrard M, Warner TD. A comparison of Marine and college women's HIV/AIDS relevant sexual behaviors. J Appl Soc Psychol 1994; 24:959-80.

14. Lerner JS, Keltner D. Fear, anger and risk. J Pers Soc Psychol 2001; 81:146-59.

Recebido em 03/Nov/2005

Versão final reapresentada em 30/Ago/2006

Aprovado em 20/Out/2006 